\title{
Communication Needs for Improved Uptake of PrEP and HIVST Services among Key Populations in Nigeria: A Mixed-Method Study
}

Olawale Durosinmi-Etti

JSI: John Snow Inc

Emmanuel Kelechi Nwala ( $\nabla$ emmanuel.nwala@gmail.com )

Population Council Nigeria

Funke Oki

NACA: National Agency for the Control of AIDS

Akudo Ikpeazu

National AIDS and STI Control Program, Federal Ministry of Health Abuja

Emmanuel Godwin

Heartland Alliance Nigeria

Paul Umoh

Heartland Alliance Nigeria

Arome Shaibu

John Snow Inc

Alex Ogundipe

NACA: National Agency for the Control of AIDS

Abiye Kalaiwo

USAID Nigeria

\section{Research Article}

Keywords: World Health Organization (WHO), key populations (KPS), PrEP and HIVST services, open data kit (ODK), geopolitical zones.

Posted Date: May 17th, 2021

DOI: https://doi.org/10.21203/rs.3.rs-427101/v2

License: (1) (1) This work is licensed under a Creative Commons Attribution 4.0 International License.

Read Full License 
Version of Record: A version of this preprint was published at AIDS Research and Therapy on November 20th, 2021. See the published version at https://doi.org/10.1186/s12981-021-00411-6. 


\section{Abstract}

\section{Background}

World Health Organization (WHO) reports that people who indulge in risky behaviours such as penile-anal sex, unprotected intercourse, multiple sex partners, and use of alcohol and illicit drugs are at risk of HIV/AIDS and have been classified as key populations (KPs). Since the introduction of PrEP and HIVST for the key population groups in Nigeria, government entities and implementing partners have used a range of channels in messaging these essential services across to the target groups - ranging from inperson, social media, television, and radio adverts. Yet, only little successes have been recorded, thereby necessitating the need to document the enabling facilitators, barriers to, and needs of the KP groups in communicating PrEP and HIVST services in Nigeria.

\section{Methods}

A mixed-method cross-sectional design was used for the study with a total of 1169 key populations in Nigeria. Surveys and qualitative exploratory methods were employed to collect data from MSM, FSWs, and key influencers of the KP groups (health providers, peer educators, HIV program officers).

Quantitative data was collected data through an open data kit (ODK), while qualitative data was collected using a topic guide in August 2020. Quantitative data analysis was conducted using SPSS version 20 for descriptive statistics while qualitative data analysis was conducted through deductive and thematic analysis based on the codebook.

\section{Results}

The KPs were mainly urban dwellers, and a majority of the participants were aged between 18 to 28yrs. However, the MSM group were of a younger population compared to the FSWs. Different level of education was reported, although a majority completed secondary education (56.1\% FSWs and $43.5 \%$ MSM). The MSM group showed more tendency to acquire higher education compared to the FSWs. For example, about $51.3 \%$ of the MSM group were undergraduates compared to $9.5 \%$ of the FSWs. The majority of the KPs were self-employed (56.4\% FSWs and 40\% MSM), however, the proportion of KPs who were unemployed was also glaring (25.8\% FSWs and $16.5 \% \mathrm{MSM})$. Only about $51 \%$ of the KPs were aware of PrEP, with typological variations (39.9\% FSWs and 62.3\% MSM). Among the MSM group, awareness about PrEP services was higher in Lagos (82.5\%), compared to $53.1 \%$ and $54.5 \%$ in $\mathrm{A} / \mathrm{lbom}$ and C/River states respectively. Among the enablers to acquiring PrEP information was the ability of the KPs to network within their communities and on personal relationships. Evidence shows that no single approach influenced the acquisition and use of PrEP information by KPs. Only about 50\% of the KPs were aware of HIVST services (40\% FSWs and 60\% MSM), although this proportion varied across the geographic locations.

The factors that enabled the acquisition and use of the prevention commodities were cross-cutting and included previous or current role as peer educator, integration of the messages, peer networking, multi- 
lingual and multi-channel presentation, use of job aids and reminders. KPs expressed the need for information on how to take PrEP, eligibility, clarification on differences between PrEP and PEP, clarification on any side effects, pricing, for PrEP, price, efficacy, sales point, dosage, available brands. A scale-up of the research across all geopolitical zones and a survey to quantify the prevalence would help in understanding the dynamics and prioritization of interventions for scaling up PrEP and HIVST services in Nigeria.

\section{Conclusions}

The policy actors should consider the preferences of the KPs and key influencers in reducing barriers to communication and increasing the uptake of PrEP and HIVST services, and ensure it is reflected in a tailored communication strategy. Since multi-linguistics and multi-channels of presentation were enablers to acquiring PrEP and HIVST messages, the communications strategy for HIV prevention should incorporate these recommendations and adapt to context-specific approaches for effective messaging.

\section{Contributions To The Literature}

- The research discussed enablers, barriers, and needs in acquiring and using effective communication for promoting Pre-exposure Prophylaxis (PrEP) and HIV self-testing (HIVST) and provides sufficient evidence to support the development of communications strategy and communication materials for key populations.

- Approaches to foster uptake of PrEP and HIVST services among key populations were highlighted and this provides lessons for other developing countries

- No one single strategy is effective in messaging PrEP and HIVST services to key populations multiple channels are likely required to message and scale up use of PrEP and HIVST services

\section{Introduction}

World Health Organization (WHO) reports that people who indulge in risky behaviours such as penile-anal sex, unprotected intercourse, multiple sex partners, and use of alcohol and illicit drugs are at risk of HIV/AIDS and have been classified as key populations (KPs) [1-3]. Though there has been progress in reducing death due to AIDS, the number of new HIV infections has been on the increase especially among the KPs, including men who have sex with men (MSM), and female sex workers (FSWs) [4-6].

There have been multiple interventions to address the burden of HIV $[1,7]$ as part of the strategy of reaching the UNAID's $95-95-95$ goals - of having 95 of people living with HIV diagnosed, $95 \%$ of diagnosed on antiretroviral therapy (ART), and $95 \% \%$ of treated virally suppressed by the year $2030 \mathrm{In}$ Nigeria, despite the introduction of pre-exposure prophylaxis (PrEP) and HIV self-testing (HIVST) services in 2017 and 2018 respectively, there is reportedly low uptake of HIV prevention services among the KPs [8-9]. Factors related to cost, literacy, language, norm, culture, type of sex work, scheduling, individual and group behavioural patterns, risk perception, perceived relevance of interventions, contextual factors 
and norms, stigma and discrimination, and constant migration among the KP groups, continue to compound low uptake of the HIV prevention services [10-14]. Addressing these barriers requires institutionalizing an effective and well-implemented communication strategy. These include - creating awareness, initiating, sustaining, and maintaining positive and desirable behaviour to prevent HIV infection among KPs.

Since the introduction of PrEP and HIVST for the key population groups in Nigeria, government entities and implementing partners have used a range of channels in messaging these essential services across to the target groups - ranging from in-person, social media, television, and radio adverts [7, 15-16]. Yet, only little successes have been recorded, thereby necessitating the need to document the enabling facilitators, barriers to, and needs of the KP groups in communicating PrEP and HIVST services in Nigeria.

The purpose of the study was to identify the communication needs and preferences of key population (KP) groups as evidence for developing strategies and interventions to increase awareness and use of HIVST and PrEP services among the KPs in Nigeria. Specifically, the study was designed to:

- To identify the enablers in acquiring and using effective communication for promoting PrEP and HIVST among KP groups in Nigeria

- To identify the barriers to acquisition and use of communication required to promote the use of PrEP, and HIVST among KP groups in Nigeria

- To describe communication needs of KPs and their support groups to promote behavior change for PrEP, and HIVST among KP groups in Nigeria

\section{Methodology}

\section{Study Sites}

Akwa Ibom, Cross River and Lagos states were chosen for this study because they priority states where the Total Market Approach for HIV Prevention Programming in Nigeria Project is being implemented. A profile of the study areas is contained in Table 1 [16-18]. 
Table 1

Profile of study states in Nigeria

\begin{tabular}{|c|c|c|c|}
\hline Indicators & Akwa Ibom & Cross River & Lagos \\
\hline HIV prevalence & $5.6 \%$ & $1.7 \%$ & $1.3 \%$ \\
\hline Population and landmarks & $\begin{array}{l}\text { South-south Nigeria, } \\
7,081 \mathrm{~km} 2 \text {, estimated } \\
\text { population of } 5,540,758\end{array}$ & $\begin{array}{l}\text { South- } \\
\text { south } \\
\text { Nigeria, } \\
20,156 \mathrm{~km}^{2} \text {, } \\
\text { estimated } \\
\text { population } \\
\text { of } \\
3,737,517\end{array}$ & $\begin{array}{l}\text { South-west Nigeria, } \\
3,577 \mathrm{~km}^{2} \text {, estimated } \\
\text { population of } \\
14,368,332\end{array}$ \\
\hline $\begin{array}{l}\text { Median age at first sexual } \\
\text { intercourse }\end{array}$ & $\begin{array}{l}17.4 \text { for women, } 22.3 \\
\text { for men. }\end{array}$ & $\begin{array}{l}17.5 \text { for } \\
\text { women, } \\
19.2 \text { for } \\
\text { men }\end{array}$ & $\begin{array}{l}20 y r s \text { for women, } \\
20.3 y r s \text { for men }\end{array}$ \\
\hline $\begin{array}{l}\% \text { of women with sexual } \\
\text { activity within the past } 4 \mathrm{wks}\end{array}$ & $42.9 \%$ & $46 \%$ & $51.2 \%$ \\
\hline $\begin{array}{l}\% \text { of women who never had } \\
\text { sexual intercourse }\end{array}$ & $15.1 \%$ & $12.1 \%$ & $20 \%$ \\
\hline $\begin{array}{l}\% \text { of men with sexual activity } \\
\text { within the past } 4 \mathrm{wks}\end{array}$ & $50 \%$ & $43.5 \%$ & $57.8 \%$ \\
\hline $\begin{array}{l}\% \text { of men who never had } \\
\text { sexual intercourse }\end{array}$ & $16.8 \%$ & $18 \%$ & $13.4 \%$ \\
\hline Fertility rate & 3.6 & 3.7 & 3.4 \\
\hline $\begin{array}{l}\text { \% of people with a } \\
\text { discriminatory attitude toward } \\
\text { people with HIV }\end{array}$ & $\begin{array}{l}61.1 \% \text { for women, } \\
38.2 \% \text { for men }\end{array}$ & $\begin{array}{l}28.8 \% \text { for } \\
\text { women, } \\
40.4 \% \text { for } \\
\text { men }\end{array}$ & $\begin{array}{l}66.9 \% \text { for women, } 65.3 \% \\
\text { for men }\end{array}$ \\
\hline $\begin{array}{l}\text { \% who reported using a } \\
\text { condom during last sexual } \\
\text { intercourse with such a } \\
\text { partner }\end{array}$ & $\begin{array}{l}33 \% \text { for females, } 72.5 \% \\
\text { for men }\end{array}$ & $\begin{array}{l}37.9 \% \text { for } \\
\text { females, } \\
65.5 \% \text { for } \\
\text { men }\end{array}$ & $\begin{array}{l}38.9 \% \text { for female, } 71.5 \% \\
\text { for men }\end{array}$ \\
\hline
\end{tabular}

\section{Study Design and Population}

A mixed-method cross-sectional design was used for the study. Surveys and qualitative exploratory methods (focus group discussion and key informant interview) were employed to collect data from MSM, FSWs, and key influencers of the KP groups (health providers, peer educators, HIV program officers).

\section{Sample Size}


The table below describes the different data collection activities for this research. The quantitative sample calculation was based on previous key population characterization in 2015 by Society for Family Health [19], from a known population size $-n=\frac{N N}{1+N N(e e) 2}$. Based on the estimated population of the KPs in each state as documented in a previous study (19), different sample size was calculated for each state (see Table 2). For the qualitative interviews, focus group discussions were conducted with the KP groups - MSM and FSWs cross the states. Also, key informant interview was conducted with KP groups, influencers, health providers, and program officers across the three states. (see Table 2 for breakdown).

Table 2

Sample distribution for quantitative and qualitative interviews

\begin{tabular}{|c|c|c|c|c|}
\hline & AVIbom & C/River & Lagos & Total \\
\hline \multicolumn{5}{|l|}{ Questionnaire administration } \\
\hline FSWs & 225 & 286 & 313 & 824 \\
\hline MSM & 143 & 99 & 103 & 345 \\
\hline Total & 368 & 385 & 416 & 1169 \\
\hline \multicolumn{5}{|l|}{ Qualitative interviews } \\
\hline FGD with MSM & 1 & 1 & 1 & 3 \\
\hline FGD with FSWs & 1 & 1 & 1 & 3 \\
\hline KIIs with MSM & 10 & 10 & 10 & 30 \\
\hline KIIs with FSWs & 10 & 10 & 10 & 30 \\
\hline KIls with KP key influencers & 17 & 17 & 15 & 49 \\
\hline
\end{tabular}

\section{Data collection and management}

Quantitative data was collected data through an open data kit (ODK), while qualitative data was collected using a topic guide in August 2020. KPs who were above 18yrs and consented to the study were included; the KPs were mobilized through their support groups, led by key influencers. Data were collected on KPs' socio-demographic characteristics, awareness of PrEP and HIVST, and preferred communication channels for PrEP and HIVST messaging. Interviews were conducted using the respondents' preferred language, recorded, and transcribed verbatim to ensure data quality. The transcripts were given unique identifiers for ease of analysis and reference.

\section{Data analysis}

Quantitative data analysis was conducted using SPSS version 20 for descriptive statistics while qualitative data analysis was conducted through deductive and thematic analysis based on the 
codebook.

\section{Results}

\section{Participants Characteristics}

The characteristics of the participants for both quantitative and qualitative interviews were comparable. The KPs were mainly urban dwellers, and a majority of the participants were aged between 18 to $28 \mathrm{yrs}$. However, the MSM group were of a younger population compared to the FSWs. Different level of education was reported, although a majority completed secondary education (56.1\% FSWs and 43.5\% MSM). The MSM group showed more tendency to acquire higher education compared to the FSWs. For example, about $51.3 \%$ of the MSM group were undergraduates compared to $9.5 \%$ of the FSWs. The majority of the KPs were self-employed (56.4\% FSWs and 40\% MSM), however, the proportion of KPs who were unemployed was also glaring (25.8\% FSWs and 16.5\% MSM) - (Table 3). 
Table 3

Participants' characteristics

\begin{tabular}{|lllll|}
\hline KP Group & FSW & \multicolumn{3}{c|}{ MSM } \\
\hline & $(\mathrm{n}=)$ & $(\%)$ & $(\mathrm{n}=)$ & $(\%)$ \\
\hline Sample size & 824 & 70.5 & 345 & 29.5 \\
\hline Age & & & & \\
\hline 18-28yrs & 526 & 63.8 & 308 & 89.3 \\
\hline 29-38yrs & 229 & 27.8 & 34 & 9.9 \\
\hline <38yrs & 69 & 8.4 & 3 & 0.9 \\
\hline Place of residence & & & & \\
\hline Rural & 304 & 36.9 & 77 & 23.3 \\
\hline Urban & 520 & 63.1 & 268 & 77.7 \\
\hline Level of education & & & & \\
\hline Primary not Completed & 60 & 7.3 & 0 & 0 \\
\hline Primary completed & 94 & 11.4 & 2 & 0.6 \\
\hline Junior secondary completed & 115 & 14 & 1 & 0.3 \\
\hline Senior secondary completed & 462 & 56.1 & 150 & 43.5 \\
\hline Advanced level & 10 & 1.6 & 6 & 1.7 \\
\hline Undergraduate & 78 & 9.5 & 177 & 51.3 \\
\hline Postgraduate & 5 & 0.6 & 9 & 2.6 \\
\hline Occupation & 76 & 9.2 & 4 & 1.4 \\
\hline Government employed & 3 & 0.4 & 9 & 2.6 \\
\hline Private sector & 18 & 2.2 & 30 & 8.7 \\
\hline Self-employed & 465 & 56.4 & 138 & 40 \\
\hline Student & 52 & 6.3 & 137 & 39.7 \\
\hline Unemployed & 210 & 25.8 & 57 & 16.5 \\
\hline Others eg retired & & & \\
\hline
\end{tabular}


The mixed-method analysis revealed disproportionate gaps in awareness about PrEP among the KPs. Only about $51 \%$ of the KPs were aware of PrEP, with typological variations (39.9\% FSWs and $62.3 \%$ MSM). The level of awareness was higher among those who previously served as peer educators to the KPs. However, there was yet some misconception around the eligibility for the use of PrEP, - for example, some KPs mistake pre-exposure prophylaxis of HIV (PrEP) for post-exposure prophylaxis (PEP). Such information asymmetry continues to limit the early introduction and use of PrEP among the KPs despite understanding the importance of PrEP in preventing HIV.

"I thought it's when you have unprotected sex or if condom breaks during sexual intercourse, then you can take the drug" LAS-KII-FSW-09

Among the MSM group, awareness about PrEP services was higher in Lagos (82.5\%), compared to $53.1 \%$ and $54.5 \%$ in $\mathrm{A} / \mathrm{lbom}$ and $\mathrm{C} /$ River states respectively. In addition, the MSM group showed a higher level of awareness of PrEP compared to the FSW group. Data shows multiple, intertwining information flow for PrEP, including peer influence, social media, NGO/CBO activities, health providers among others. MSM group was likely to acquire PrEP information via peer influence $(46 \%)$ and social media $(14.4 \%)$ compared to the FSW group (24.2\% and 6.1\%) respectively. Contrarily, FSWs reported higher proportions of $\mathrm{NGO} / \mathrm{CBO}$ and health provider influence (usually face-to-face messaging) than the MSM group across the three states.

Among the enablers to acquiring PrEP information was the ability of the KPs to network within their communities and on personal relationships. Information about PrEP continues to diffuse among the KPs - some community members reported receiving or giving out information on PrEP and HIVST to other community members who do not participate in the peer sessions or who are not in the closed community groups. MSM group demonstrated higher level of awareness and were more likely to network information on PrEP than FSWs.

\section{Current Channels for Messaging PrEP Services:}

Evidence shows that no single approach influenced the acquisition and use of PrEP information by KPs. However, the Face-to-face channel was the most reported means of acquiring PrEP information (32.6\% FSWs and $49 \%$ MSM). Varying media channels have been explored by KPs to acquire PrEP information WhatsApp, Facebook, Instagram and Twitter, and short message service (SMS). However, the use of mixed languages - local dialect, pidgin and English languages for messaging PrEP across the three states enabled acquisition and use of such information. Yet, there are still other channels such as manjam, tindre and grindr that are reportedly exclusive to only community members, providing more secure, encrypted opportunities for KPs to express themselves and share information with their key influencers.

"Yes, Instagram, Facebook, we have even some Apps such as manjam, tindre and grindr that are strictly for community members". LAS-KII-PM-02. 
"I get the information from the internet, mass media. There are even groups on WhatsApp, Facebook that you can belong to and they talk about it (PrEP)" AKS-FGD-MSM-P1

Complimentarily, health providers reported the use of critical job aids to facilitate PrEP and HIVST awareness within KP communities irrespective of typology.

"We go with posters and even fliers, we show them all those things" LAS-KII-HCP-03

\section{Awareness about HIVST Services:}

Gaps exist in awareness about HIVST, especially among the FSWs. Only about $50 \%$ of the KPs were aware of HIVST services (40\% FSWs and 60\% MSM), although this proportion varied across the geographic locations. For example, among the MSM group, awareness was higher in Lagos $(71 \%)$ compared to A/Ibom (57\%) and C/River (52\%) states respectively. FSWs who indicated some level of awareness around HIVST acquired it mainly through a face-to-face peer session. However, many KPs did not have a good understanding of the self-testing process.

Mixed reactions from the respondents suggested that even though there has been an effort to increase HIVST awareness, there may have been slow progress in the uptake of the services.

"For now, we just only have the awareness HIVST, but we have not started providing it" LAS-KII-MSM-03

"I don't know about it before now, but If I see it, I can use it on myself" LAS-KII-FSW-01

\section{Current Channels for Messaging HIVST Services}

For HIVST messaging, the in-person channel was the most reported ( $85 \%$ FSWs and $68 \% \mathrm{MSM}$ ). This trend was followed by social media channels, including Facebook, Twitter, and Instagram ( $9.4 \%$ FSWs and $31.6 \% \mathrm{MSM}$ ). These proportions varied across the study locations. However, the importance of participatory learning and action platforms such as FGDs cannot be overemphasized. FGDs indicated that HIVST was newly introduced to the KP community groups, and integrated with other commodities such as PrEP, condoms, and lubricants, given that they all target the same population groups.

Qualitative data indicated that some MSM reported that they became aware of HIVST initially during peer group discussion and subsequently through NGOs, social media, flyers, emails, and face-to-face with a health care provider. While many of the respondents did not have a good understanding of the workability of the self-testing process, there were selected few who could properly describe the procedures. This finding of the key population's inability to describe procedures for self-testing was not encouraging especially in spite of using some KPs as ambassadors for promoting HIVST services. This might also explain the low access of the KPs to the self-testing services.

"The information I heard is that the HIV self-testing is a kit been used personally in your own convenient time to test yourself either from oral, oral fluids or from blood, so, so that's the information I got from the HIV test" CRS-KII-MSM-07

Page $11 / 28$ 
"Well, because a lot of our community members are not aware of the reason why they should use HIVST some of those people that we invite usually come for the PrEP services, although the number is not that many, is not that high" LAS-KII-PM-01

Cross-sectional views of the respondents suggested that a variety of channels are being used to encourage wider communication of HIVST information, indicating that there is no one channel for effective delivery of the information.

"We use Facebook, we use WhatsApp, we use Instagram, we use Twitter". LAS-KII-PM-01

"I belong to a group on Facebook where I get HIV messages from and that is how I got to know of the PrEP we are also talking about it now" CRS-FGD-MSM

In line with the COVID-19 social distancing protocol, program managers reported that the WhatsApp and other social media handles such as Facebook, Instagram, and Twitter handle also act as social support groups which they use to upload videos displaying information and demonstrating how to and how not to conduct self-test for HIV.

\section{Enablers to Acquisition and Use of PrEP and HIVST Messaging among the KPs}

The factors that enabled the acquisition and use of the prevention commodities were cross-cutting and are presented in Table 4 below:

Table 4

Enablers to Acquisition and Use of PrEP and HIVST Information among the KPs

\begin{tabular}{|c|c|c|}
\hline Enablers & PrEP & HIVST \\
\hline Participation in peer education sessions/previous or current role as peer educators & $\mathrm{x}$ & $\mathrm{x}$ \\
\hline Integration of the messages with other commodities & $\mathrm{x}$ & $x$ \\
\hline Removal of user fees for PrEP & $\mathrm{x}$ & ( \\
\hline Community linkages/networking - especially among MSM & $\mathrm{X}$ & $\mathrm{X}$ \\
\hline Multi-lingual presentation of the messages & $x$ & $x$ \\
\hline $\begin{array}{l}\text { Use of diverse channels for peer sessions - face-to-face, social media (Facebook, } \\
\text { Instagram, and WhatsApp) }\end{array}$ & $\mathrm{X}$ & $x$ \\
\hline Use of job aids and experiential learning approaches & $\mathrm{X}$ & $x$ \\
\hline Reminders via phone call or SMS & $\mathrm{X}$ & प \\
\hline \multicolumn{3}{|l|}{ Outreach program } \\
\hline Note: $X=$ presence of enabler; $\mathbb{X}=$ absence of enabler & & \\
\hline
\end{tabular}




\section{Barriers to Acquisition and Use of PrEP and HIVST Messages}

\section{Stigma}

Findings in this study indicated that stigma includes self and social stigma. The initial self-stigma comes due to the level of literacy required to participate in the social media-based peer session, retrieving messages, and navigating through the platforms. This phenomenon is preponderant among FSWs than MSM and leads to KPs pairing with their friends to receive social media/device-based information on the prevention commodities.

"Some people don't know how to read and write - some of them will choose to join their friends to do it because their friends are doing it. Some will feel ashamed and isolate themselves because they can't read" LAS-KII-FSW-07

From the KPs and program managers' point of view, there is the tendency that some KPs who test positive for HIV may commit suicide due to social stigma and in a bid to reject the reality of the test results. Also, health provider perspectives indicated that KPs feel stigmatized among their peers for using PrEP.

"You know some persons can be funny. We don't want a situation whereby somebody will test his or herself and commit suicide" LAS-KII-FSW-09

"There are people who may not be comfortable with your sexual orientation and these commodities, because of this kind of situation, you can't hide like that. So, you have to tell the truth" AKS-KII-MSM-04

\section{Discrimination}

There is reported discrimination and harassment of KPs by security agencies in Nigeria and the neighbourhood. This is one of the reasons the KPs especially MSM, avoid face-to-face community activities where they could learn about PrEP, HIVST, and other prevention approaches. Discrimination sometimes hinders the MSM group to retain peer discussions and community-related information on their phones due to security personnel interferences to search people's devices.

"When a policeman sees some of us walking like a girl, all you'll hear is "identify yourself". And maybe you don't have an ID card, the policeman will go to your phone and before you know it he'll abuse and infringe into your right just because of your physical appearance". LAS-KII-PM-02

The purported harassment, especially of the MSM community by government authorities was highlighted as one that will require assurance for confidentiality if they must receive face-to-face information.

"Face to face is good but depends on what information you're sharing. There is something about our community, we need assurance that when they gather another thing will not happen" LAS-FGD-MSM 


\section{Fear}

The fear of social embarrassment and pain of being associated with the use of ARV for HIV leads to withdrawal, rejection, and missed opportunities to start-up PrEP among the KPs. There is the concern that their partners and the entire society may inflict emotional and social injuries when found with medicines for PrEP. Some KPs opined that they can trade off life instead of taking PrEP.

"It is just fear - you hear KPs say that they don't want a situation where they will take it because people believe that it is only those that already have HIV infection that supposes to take ARVs- that is their fear" LAS-KII-HCP-01

"Because they will be scared of information, some people believe they should die than knowing it (their status) which will cost them so much" LAS-KII-MSM-05

\section{Privacy}

The absence of privacy was found to limit the acquisition of information on PrEP and HIVST services. A significant number of the study participants do not have employment and share accommodation and personal effects with other household members. Therefore, they do not have privacy in keeping and using these essential HIV prevention commodities. Some MSM feel that closed groups such as WhatsApp and Facebook may be abused with gossip and intangible discussions, leading to a lack of willingness of some community members to participate in the discussions.

"The reason, I don't subscribe to this WhatsApp of a thing is, in the beginning, it would be very interesting but at the middle and the end, it's all gossips and stuff that make it boring and uninteresting" LAS-FGD$M S M-P 3$

Nevertheless, health providers reiterated that KPs tend to be more worried about the confidentiality of the test results, especially when a facility-based confirmatory test is conducted. From the peer educators' perspective, one major challenge in relaying information on HIVST is the perceived breach of privacy. For example, peer educators have witnessed KPs' anxieties around how to communicate positive test results to their influencers who may want to refer them to health providers at the facilities for a confirmatory test.

"Even if they take the self-testing kit to their house to test themselves and they come out positive, they will not come out to the facility to tell them you are positive" LAS-KII-PE-01

Also, KPs opined that the challenge of peer sessions is that since it's a group-based event, some KPs may not open up, thinking that there may be information diffusion to wrong personalities.

\section{Limited access to channels of communication}

Despite the KPs reporting that logistics was a huge challenge for face-to-face approaches, program managers rather suggested more cost-effective approaches - social media. Although there were positive responses regarding messaging through social media, some KPs complained that they do not have 
smartphones required to access social media interactions. Some of the FSWs complained about losing the social media handle addresses, mostly Instagram, and Twitter, while some have never been on social media due to lack of smartphones.

"For now, I am not on any social media platform because I don't have an android phone, because they stole it" LAS-KII-FSW-01

MSM who preferred social media-based channels were of opinion that social media-based communication messages could be misinterpreted, probably due to the inability to correctly interpret the PrEP or HIVST messages. However, KPs recognized that social media benefits them in terms of cost and time savings as well as having to worry less to dress up to attend face-to-face sessions.

\section{Limited electric power supply and internet access}

Frequent low phone battery (resulting from limited power supply) and limited internet access were among the potential barriers to effective communication of PrEP and HIVST services among the FSWs especially as it has to do with social media platforms. For those KPs who participate in the social media-based peer sessions, electricity outages and battery discharge sometimes limit their ability to effectively participate and receive information via online peer sessions.

"Access to internet data can be a barrier because when there is no data, I can't use Facebook or WhatsApp" AKS-KII-FSW-01

"Now when I am talking about WhatsApp or Facebook, the only thing that can hinder me from getting the information might be when I don't have internet data. If I don't have data, I cannot participate to get the information" AKS-KII-MSM-09

\section{Language of communication}

Some KPs reported that if PrEP and HIVST messages are presented in languages that they are not conversant with, the goal of the communication may be defeated. KPs insisted that those in rural settings should be considered when designing PrEP and HIVST messages in terms of tailoring the languages to a dialect that people would understand.

\section{Socio-economic issues}

Several of the MSM are unemployed and depend on their families who may not be able to afford the costs or may not want to pay for PrEP and HIVST services. Poor socio-economic status of KPs may limit attention span during online peer sessions and may lead to inability of the KPs to invest internet data and smartphones to participate in online discussions. Arguably, key influencers need to stay online for longer hours, require data to upload and maintain social media statuses and provide feedback and follow-up services to KPs who may be in need. 
From the key influencers' and program managers' point of view, costs associated with participating in peer sessions (including in-person, virtual, and SMS) may hinder the communication of PrEP and HIVST information - these costs are usually indirect and could include costs of refreshment and transportation to the venue. Some KPs argued that the direct cost of PrEP and HIVST may deter KPs from accessing the services, especially for MSM who may be out of a job or depend on the caregivers for support if services are not provided free of charge.

"After approaching them, talking to them and showing them how to use it, the price for getting PrEP and HIVST should not be too high so that people can afford it" LAS-KII-FSW-01

KP influencers reported limited access to health facilities for referral, coupled with a need for follow-up on the use of the kit, especially in circumstances where the client tests negative and needs to be placed on PrEP or positive and requires further HIV care and treatment.

For non-face-to-face peer sessions, money is necessary to purchase a data bundle to enable KPs to receive social media-based PrEP and HIVST information. Some KPs reported that if the distance to the health facility is very far, they may not want to go there all the time due to indirect costs associated with transportation. For key influencers, lack of finance to procure data and other resources was reported as a challenge to relaying PrEP and HIVST information to KPs. Arguably, key influencers need to stay online for longer hours, require internet access to upload and maintain social media statuses and provide feedback and follow-up services to KPs who may be in need.

Program managers think that PrEP and HIVST information dissemination is impeded by the socioeconomic status of the KPs. For example, poverty and hunger may limit attention span during peer sessions and may lead to the inability of KPs to purchase data and smartphones to enable them to participate in online discussions. Similarly, key influencers reiterated that the high cost of the commodities limits communication of PrEP and HIVST information to the KPs, especially the MSM community. This is because most MSMs are unemployed and depend on their families who may not be able to afford the costs or may not want to pay for the services. Aside costs, health providers anticipate that lack of incentives impede PrEP and HIVST messaging, arguing that KPs are attracted by nonfinancial rewards - whether through an online meeting or face-to-face.

"I know that Prep is very expensive, so, it is not easy to get" LAS-KII-HCP-02

\section{Inadequate inclusion of KPs in program planning and implementation}

There were also complaints that KPs and their key influencers are excluded from program planning including initial and evaluation stages. Key influencers think that incorporating KPs and KPIs opinions would enrich the program's success as it relates to increasing awareness for the uptake of preventive services.

But again, there were views that there are not enough champions to drive the awareness campaign for PrEP and HIVST. This is worsened by the prohibition laws in Nigeria which put peer educators at risk of 
manhandling by the police and hoodlums.

\section{Poor feedback mechanism}

Influencers complained that KPs do not always return with the feedback of their HIV self-test results, making it difficult to follow up with further PrEP and other HIV services.

"So, the major challenge with self-test kit is that most often when they access this service, they don't always come back with feedbacks" LAS-KII-KPI-01

\section{Poor Attitudes of Healthcare Providers}

Key informants interviewed indicated that KPs experience disrespect from providers, especially when the provider is not a community member, ranging from emotional abuse to delays, denial of services, and a bridge of confidentiality and privacy. KPs opined that poor interpersonal communication behaviors of health providers can drive them away from accessing PrEP and HIVST services or even from attending or following up with services.

Character, it goes a long way, the way the health providers welcome clients, counsel and interact has a lot to do with health-seeking" CRS-KII-FSW-07

"Sometimes, the health providers talk anyhow to clients. The way you talk to me will make me decide if I want to come back again" CRS-KII-MSM-01

\section{Lack of incentives}

KPs often expect financial and other non-financial rewards before participation in information sharing sessions, especially when it involves in-person peer sessions. Non-financial incentives included the provision of free condoms, lubricants, and HIVST kits while financial reward relates to transport fees despite that peer educators visit the KP locations, especially for the FSWs.

"You know here in Nigeria if you want to get somebody's mind, you're supposed to go with something, so there must be something like an incentive to attract them" LAS-KII-HCP-03

"Sometimes, when we come for the meetings (peer sessions), you guys don't give us condoms or anything at all" CRS-KII-FSW-09

\section{KPs attitudes and perceptions}

The indifference of some KPs also limit the acquisition of PrEP and HIVST information, coupled with concerns of why PrEP has to be taken daily and HIVST conducted routinely. These concerns have also led to discontinuations of PrEP use. Among some KPs, there are still doubts about the efficacy of PrEP in protecting an individual, occasioned by advocacy for the combined use of PrEP and condoms for effective protection. KPs reported that this tends to be cumbersome in practice but would rather continue using condoms than PrEP. 


\section{Communication Needs of KPs for PrEP and HIVST Services in Nigeria}

\section{Receptiveness to future PrEP and HIVST messages}

Quantitative data indicated that about 95.5\% FSWs and 88.7\% MSM indicated that they need to receive PrEP messages in the future. Similarly, $94.2 \%$ FSWs and $86.7 \%$ MSM were willing to receive HIVST messages in the future. Both FGDs and KIls elicited reasonable and extreme willingness to receive more information on PrEP and HIVST regardless of the typology of KP. Despite a lack of awareness about PrEP and HIVST, KPs are willing to embrace the messages and are also eager to disseminate PrEP and HIVST information within and across their networks.

"I like it, if they want us to participate, I am ready" LAS-KII-FSW-01

"I need to have more knowledge about it so that I can relate the information to others" AKS-KII-MSM-09

Most KPs want more information on how to take PrEP, eligibility, clarity on the differences between PrEP and PEP, side effects, and pricing for PrEP.

"For now, I don't know anything about the drugs, but if I know more about the drugs (PrEP) I will communicate with my friends and anybody around me. But for now, I don't know anything about it" LASKII-FSW-07

\section{Preferences for channels of communication}

The preferred channels to receive PrEP and HIVST messages varied across KP typologies. While the FSWs preferred mainly face-to-face, phone calls, and SMS, the MSM group would prefer social mediabased channels - mainly WhatsApp, Facebook, Instagram, and Twitter. Also, MSMs advocated for other closed social media channels that only admit community members who are usually introduced by their peers, including Mangam, Tindre, Grindre. The main reason MSM prefers social media approach to faceto-face is because their sexual orientation is criminalized, coupled with stigma and discrimination around it. There may be a high attrition rate in response to the physical meetings. This attribute is complicated by the high mobility of members of the MSM community.

"In our community, we are very mobile, almost every community member you see out there is on social media because that's where you meet new people, that's where we interact, that's where people socialize and meet themselves" LAS-KII-MSM-08

For some of the FSWs, reasons for not preferring social media channels included the unavailability of smartphones for chatting. For KPs who preferred face-to-face channels, the reason was that it offers the opportunity to ask questions, receive instant feedback, and facilitates understanding, while watching nonverbal cues. SMS was also not ranked top priority owing to the assumption that the receiver might decide 
to ignore the messages for a couple of days or may delete them permanently without reading them. SMS might also be subject to misinterpretation.

"I like WhatsApp. I like Instagram, I like Facebook also". LAS-KII-FSW-05

"Yea, face to face, at least you will understand better" LAS-KII-MSM-03

"Because it is confidential, so I prefer WhatsApp, nobody has access to my WhatsApp and see what I am chatting" AKS-KII-MSM-09

"Text messages, WhatsApp, face-to-face, Instagram, Facebook, but I prefer text messages". Text messages as most of us don't have smartphones LAS-KII-FSW-06

The expected frequency for receipt of PrEP and HIVST information varied among the respondents, ranging from daily, weekly to monthly basis. There was no consistent pattern and time to receive HIV preventive messages.

Brothel-based FSWs would prefer that their chair ladies act as peer educators, enabled with posters, handbills, and other communication materials that will improve their work. Despite issues of not having a smartphone for social media-based communication, FSWs think that social media or telephone might confuse them and would rather prefer face-to-face interactions to receive PrEP and HIVST messages as this would offer opportunities for clarifications.

"I prefer it because I will be seeing the person explaining it to me, the way he's explaining it on the phone I might be confused, and I might not know what to do so I prefer face to face" LAS-KII-MSM-01

"I will like the person to talk to me, one-on-one interaction because I don't have a smartphone now. Also, I would like to receive text messages on it" LAS-KII-FSW-06 
Table 5

Preferred channels of communication for PrEP and

HIVST among KPs

\begin{tabular}{|lllll|}
\hline & PrEP & & HIVST \\
\hline & FSW & MSM & FSW & MSM \\
\hline Face-to-face & 67.9 & 59.2 & 67.5 & 47.8 \\
\hline WhatsApp & 8.3 & 17.6 & 9.3 & 20.9 \\
\hline Social media & 0.3 & 1.3 & 5.4 & 24.6 \\
\hline SMS & 20.1 & 12.4 & 24.6 & 20.6 \\
\hline Poster/flier & 0.8 & 1 & 4.8 & 1.8 \\
\hline Radio/television & 1.9 & 1.3 & 6.3 & 3.5 \\
\hline Other & 0.9 & 7.2 & 1.7 & 6.4 \\
\hline
\end{tabular}

For MSMs, the most preferred medium for meeting with a health provider is KP-specific platforms and channels, including the one-stop shops (OSS) that are community-driven and friendly.

"But for me, I think I prefer an NGO that deals with the key population, you don't need to be asked unnecessary questions compared to the general hospitals except in terms of STI" CRS-FGD-MSM.

KPs recommended a disaggregated, targeted communication for wider coverage, and also think that PrEP and HIVST information can be disseminated through churches since almost everyone attends religious gatherings. For instance, while those with smartphones can join social media, others without smartphones can be encouraged to attend face-to-face sessions. Face-to-face was repeatedly to better understanding, the opportunity for clarifications, and deeper interaction with the counsellor/peer educator. Consistently among FSWs, there were concerns that social media discourages the active participation of less-educated community members.

\section{Communication with healthcare providers}

There was no definite approach to meeting or consulting with health providers identified in this study. Some KPs preferred meeting their health providers face-to-face, though with flexibility for other channels of communication, mainly due to the non-verbal cues that face-to-face channel offers in supporting understanding for sensitive health issues or symptoms. However, there are strong indications for telemedicine among some KPs.

"They can call me on the phone if I need anything, I will tell them to come and meet me here" LAS-KII-FSW01 
"WhatsApp messages are perfect because it's convenient. Not seeing doctors face to face. Even when I travel to the village, I can still get my message via WhatsApp without issues". LAS-KII-FSW-03

"I prefer face to face because I would be able to ask questions and I will be free to express myself. Like you know, it's just me and you here, people are not much in this place. If people were to be much here, I would not be able to answer all these questions or seat here to gist with you f" AKS-KII-FSW-03

Among the FSWs, preference for health care providers varied - while some FSWs prefer to meet older male health providers, some FSWs prefer to consult middle-aged female health providers who they perceived would maintain confidentiality for the care they provide.

"I need a mature male provider because they can understand. You can never hear your secret from someone else - a mature person can keep your secret" LAS-KII-FSW-03

Expected future content of the PrEP and HIVST messaging: KPs expressed the need for information on how to take PrEP, eligibility, clarification on differences between PrEP and PEP, clarification on any side effects, pricing, for PrEP, price, efficacy, sales point, dosage, available brands. However, Most KPs preferred to received PrEP and HIVST information in multiple languages: English Language or pidgin English, or local dialect.

\section{Discussion}

Given the low level of awareness about the HIV prevention services (PrEP and HIVST) among the KPs found in this study, strategic planning is required to ensure increased access to PrEP and HIVST information so as to support Nigeria's pursuit to reduce incidence of new HIV infection. This finding about low level of awareness was not surprising since Nigeria is yet to roll out a national strategic plan that will enhance awareness and demand generation for PrEP and HIVST services. Cross-cutting factors that may encourage an implementation of sustainable and targeted strategies that are KP-friendly to acquiring PrEP and HIVST messages were elicited from the study. The findings suggest that dissemination of PrEP and HIVST messages will require the use a targeted messaging and disaggregated audience, hence, content should consider the preferences of the KP groups for effective and sustainable impacts [20-21]. Emphasis should be laid on the specific channels that best attract the attention of the KP groups in dispelling misconceptions, stigma, discrimination, fears, and other barriers that may be contextual to increase the acquisition and use of PrEP and HIVST messages. These findings also suggest that HIV prevention program managers should design a well-tailored national communication strategy with inputs from all key stakeholders including service providers, and end-users [22].

Findings from the key influencers corroborated the thoughts of the key population groups concerning the barriers to acquisition and the use of information to promote PrEP and HIVST behaviour change communication. However, this has been similarly reported in other studies in Nigeria and elsewhere [2, 17, 23]. It was not surprising to find that stigma, shame, discrimination limit the acquisition and use of HIV prevention messaging in the study areas. This might be because, in Nigeria and Africa, individual 
behaviours are largely driven by religion and culture, thereby possibly instigating self-stigma and shame. It is, therefore, necessary to consider these barriers when designing a robust communication strategy that will support increased awareness and access to PrEP and HIVST services [9] while leveraging ongoing cross-cutting strategies to eliminating the barriers.

From this study, KPs can strongly network to increase awareness and access to PrEP and HIVST services in Nigeria, as applied to other settings [20-21]. This is quite encouraging considering the importance of peer influence in producing multiplier effects in behaviours. This will continue to make the peers receptive to the interventions and help reach other peers in their network - especially the hard-to-reach. However, this idea reasonably calls for an opportunity to engage some KPs as champions to increase access to the hidden KPs. Having established that a total market approach is a vital mechanism to getting the KPs to pay for PrEP and HIVST services [6, 22-23], the findings on the receptiveness of KPs to receive PrEP and HIVST messages in the future raises high hopes and corroborates with previous studies [24] since the KPs reported initially receiving messages via multiple channels. Multiple channels including social media will provide avenues for programmers and key influencers to reinforce messages on future financing plans for PrEP and HIVST when necessary.

This study found that KPs who visit OSS or whose friends visit OSS were more likely to be aware of PrEP than other community members. Therefore, health care providers and key influencers should be ready to provide all-encompassing messaging that will lead to positive behavior change. The contents of the future PrEP and HIVST messaging should reflect the views of the KPs in terms of access points, pricing, efficacy, available brand among others $[8,15,25]$.

\section{LIMITATIONS}

This study was formative and conducted only in three states of the federation - Akwa lbom, C/River, and Lagos and did not represent opinion across the six geopolitical zones. A scale-up of the research across all geopolitical zones and a survey to quantify the prevalence would help in understanding the dynamics and prioritization of interventions for scaling up PrEP and HIVST services in Nigeria.

During the data collection period, it was difficult to reach the non-brothel based FSWs to ensure a wider opinion. Therefore, further research should be conducted to elicit preferences of the non-brothel based KPs and ensure it reflects in the national communication strategy.

\section{Conclusion}

The policy actors should consider the preferences of the KPs and key influencers in reducing barriers to communication and increasing the uptake of PrEP and HIVST services, and ensure it is reflected in a tailored communication strategy. Since multi-linguistics and multi-channels of presentation were enablers to acquiring PrEP and HIVST messages, the communications strategy for HIV prevention should incorporate these recommendations and adapt to context-specific approaches for effective messaging. Understanding that peer influence is essential in propagating PrEP and HIVST messages, program 
managers should continue to explore avenues that produce multiplier effects in behaviour change in rolling out the tailored communication strategy while maintaining the confidentiality of information and purpose. Other concurrent and parallel activities that are targeted at increasing uptake of PrEP and HIVST information must be aligned to the national communication strategy for ease of tracking, reporting, and reinforcement. Considering the strong networking effects of KPs in the dissemination of communityrelated information, programs should continue leveraging the KPs as ambassadors, peer educators, and service providers, especially in linking the hard-to-reach groups within their networks, while considering $\mathrm{KP}$ typologies.

\section{Abbreviations}

WHO - World Health Organization

HIV - Human Immuno-deficiency Virus

AIDS - Acquired Immune Deficiency Syndrome

KPs - Key Populations

MSM - Men who have sex with men

FSWs - Female sex workers

ART - antiretroviral therapy

PrEP - Pre-exposure prophylaxis

HIVST - HIV self-testing

ODK - Open data kit

SPSS - Statistical Package for Social Sciences

NHREC - National Health Research Ethics Committee

JSI - John Snow Incorporated

\section{Declarations}

\section{Ethical approval and consent to participate}

The research protocol was approved by the National Health Research Ethics Committee of the Federal Ministry of Health (NHREC/01/01/2007-28/08/2020) as well as the Health Research and Ethics Committee of John Snow, Inc (JSI) Institutional Review Board (IRB). Verbal and written consent was sought from each of the study participants and duly documented. 


\section{Consent for publication}

Not applicable

\section{Availability of data and materials}

All datasets used and/or analysed for this study are available on request from the corresponding author. They are currently safely stored at JSI archive.

\section{Competing Interest}

The authors declare that there is no competing interest

\section{Funding}

This project was supported by USAID under sub-award by Heartland Alliance LTD/GTE under the Key Populations CARE 1 Project. The funding source had no role in study design; data collection, analysis, or interpretation; writing the report; or the decision to submit the report for publication. Its contents are solely the responsibility of the authors and do not necessarily represent the official views of the USAID.

\section{Authors' Contributions}

$\mathrm{OD}$ and EKN designed this study protocol with significant input from FO, Al and AS. EG, PU, and AO. AK contributed importantly to the theoretical and practical considerations of study implementation and critically evaluated study procedures. EKN drafted the manuscript and was the corresponding author. All authors reviewed, critically revised, and approved the final version of this manuscript prior to submission.

\section{Acknowledgments}

We acknowledge all stakeholders (professionals, implementing partners, and civil society organizations (CSOs), who provided valuable inputs during the study. We acknowledge USAID for funding this study through sub-award by Heartland Alliance LTD/GTE under the Key Populations CARE 1 Project, national agency for the control of AIDS (NACA), and national AIDS and STIs Control Program (NASCP) for providing leadership and an enabling environment. Nevertheless, we further acknowledge the respondents who offered their time and provided valuable information during the study.

\section{Authors' information}

Not applicable

\section{References}

1. World Health Organization. Consolidating guidelines for HIV prevention, diagnosis, treatment, and care for key populations. WHO 2014. https://www.who.int/hiv/pub/guidelines/keypopulations/en/ 
Assessed 26th September 2020.

2. Wolf RC, Bingham T, Millett G, Wilcher R. Optimizing the impact of key population programming across the HIV cascade. Journal of the International AIDS Society, 2018 (21) 5.

3. Reisner SR, Mimiaga MJ, Skeer M, Mayer KH. Beyond Anal Sex: Sexual Practices Associated with HIV Risk Reduction among Men Who Have Sex with Men in Boston, Massachusetts. AIDS Patient Care STDS. 2009 Jul; 23(7): 545-550..

4. Nigeria HIV/AIDS. Indicator and Impact Survey (NAIIS) 2019. Federal Ministry of Health.

5. Ochonye B, Flayan MO, Fatusi AO, Bello BM, Ajidagba B, Emmanuel G, Umoh P, Ayo Y, Jaiyabo T. Sexual practices, sexual behavior, and HIV risk profile of key populations in Nigeria. BMC Public Health. 2019;19:1210.

6. Risher K, Meyer K, Beyrer C. The HIV treatment cascade in men who have sex with men, people who inject drugs, and sex workers. Curr Opin HIV AIDS. 2015 Nov;10(6):420-9.

7. Tun W, Vu L, Dirisu O, Sekoni A, Shoyemi E, Njab J, Ogunsola S, Adebajo S. Uptake of HIV self-testing and linkage to treatment among men who have sex with men (MSM) in Nigeria: A pilot program using key opinion leaders to reach MSM. J Int AIDS Soc. 2018 Jul; 21.

8. World Health Organization. Guidelines on HIV Self-Testing and Partner Notification: Supplement to Consolidated Guidelines on HIV Testing Services. WHO; 2016.

https://www.ncbi.nlm.nih.gov/books/NBK401675/. Accessed 26th September 2020.

9. Nwaozuru U, Iwelunmor J, Ong JJ, et al. Preferences for HIV testing services among young people in Nigeria. BMC Health Serv Res. 2019;19:1003.

10. Musinguzi N, Kidoguchi L, Mugo NR, et al. Adherence to recommendations for ART and targeted PrEP use among HIV serodiscordant couples in East Africa: the "PrEP as a bridge to ART" strategy. BMC Public Health. 2020;20:1621 (.

11. Sun L, Liu A, Li J, et al. Is PrEP necessary during natural conception in HIV-1-serodiscordant couples on ART with suppressed viral load? A retrospective cohort analysis. BMC Infect Dis. 2020;20:195 (.

12. Ogunbajo A, Iwuagwu S, Williams R, Biello K, Mimiaga MJ. Awareness, willingness to use, and history of HIV PrEP use among gay, bisexual, and other men who have sex with men in Nigeria. PLoS One. 2019;14(12).

13. Ogunbajo A, Kang A, Shangani S, Wade RM, Onyango DP, Odero WW, Harper GW. Awareness and Acceptability of pre-exposure prophylaxis (PrEP) among gay, bisexual and other men who have sex with men (GBMSM) in Kenya. AIDS Care. 2019 Oct,31(10):1185-1192.

14. Njau B, Covin C, Lisasi E, et al. A systematic review of qualitative evidence on factors enabling and deterring uptake of HIV self-testing in Africa. BMC Public Health. 2019):;19:1289 (.

15. World Health Organization. World Health Organization. HIV self-testing strategic framework: a guide for planning, introducing, and scaling up. Geneva: World Health Organization; 2018. License: CC BYNC-SA 3.0 IGO. 
16. National Population Commission (NPC) [Nigeria] and ICF. Nigeria Demographic and Health Survey 2018. Nigeria: NPC and ICF: Abuja; 2019.

17. Emmanuel G, Folayan M, Undelikwe G. Ochonye B, Jaypba T, Aiwonodagbon B, Bilali C, Umoh P, Ojemeiri K, Kalaiwo A. Community perspectives on barriers and challenges to HIV pre-exposure prophylaxis access by men who have sex with men and female sex workers access in Nigeria. BMC Public Health. 2020;20:69.

18. Oladele DA, Gab-Okafor CV, Oke B, Kalejaiye OO, Somefun EO, Ezeobi PM, Gbajabiamila T, Adu RA, Onwujekwe DI, Ohwodo H, Odubela O, Odunukwe NN, David N, Ezechi OC. Pattern, challenges and correlates of condom use among Nigerians living with HIV infection. Asian Pac J Trop Biomed. 2014; 4(1): 198-203.

19. Ezirim IO, Ogungbemi K, Daniel U, Blanchard J \& Ezire O. Evidence from epidemic appraisals in Nigeria. African Evaluation Journal 2015; 3(2).

20. Figueroa C, Johnson C, Verster A, Baggaley R. Attitudes and Acceptability on HIV Self-testing Among Key Populations: A Literature Review. AIDS Behav. 2015; 19(11): 1949-1965.

21. Vara PA, Buhulula LS, Mohammed FA, Njau B. Level of knowledge, acceptability, and willingness to use oral fluid HIV self-testing among medical students in Kilimanjaro region, Tanzania: a descriptive cross-sectional study. AIDS Res Ther. 2020;17:56.

22. World Health Organisation. Who strategic communications framework for effective communications. WHO. File:///C:/Users/Emma/Downloads/communication-framework.pdf. Accessed on October 10, 2020.

23. Gombe MM, Cakouros BE, Ncube G, Zwangobani N, Mareke P, Mkwamba A, et al. (2020) Key barriers and enablers associated with uptake and continuation of oral pre-exposure prophylaxis (PrEP) in the public sector in Zimbabwe: Qualitative perspectives of general population clients at high risk for HIV. PLOS ONE 15(1).

24. Taggart T, Grewe ME, Conserve DF, Gliwa C, Roman Isler M. Social Media and HIV: A Systematic Review of Uses of Social Media in HIV Communication. J Med Internet Res. 2015:17(11).

25. FHI360/LINKAGES Nepal, Report of Exploring the Uptake and Acceptability of HIV SelfTesting for men who have sex with men, male sex workers, and Transgender people in Nepal. 2018, Kathmandu, Nepal, FHI 360/LINKAGES Nepal.

https://www.fhi360.org/sites/default/files/media/documents/resource-nepal-hivst-report.pdf. Accessed March 21, 2021.

\section{Figures}




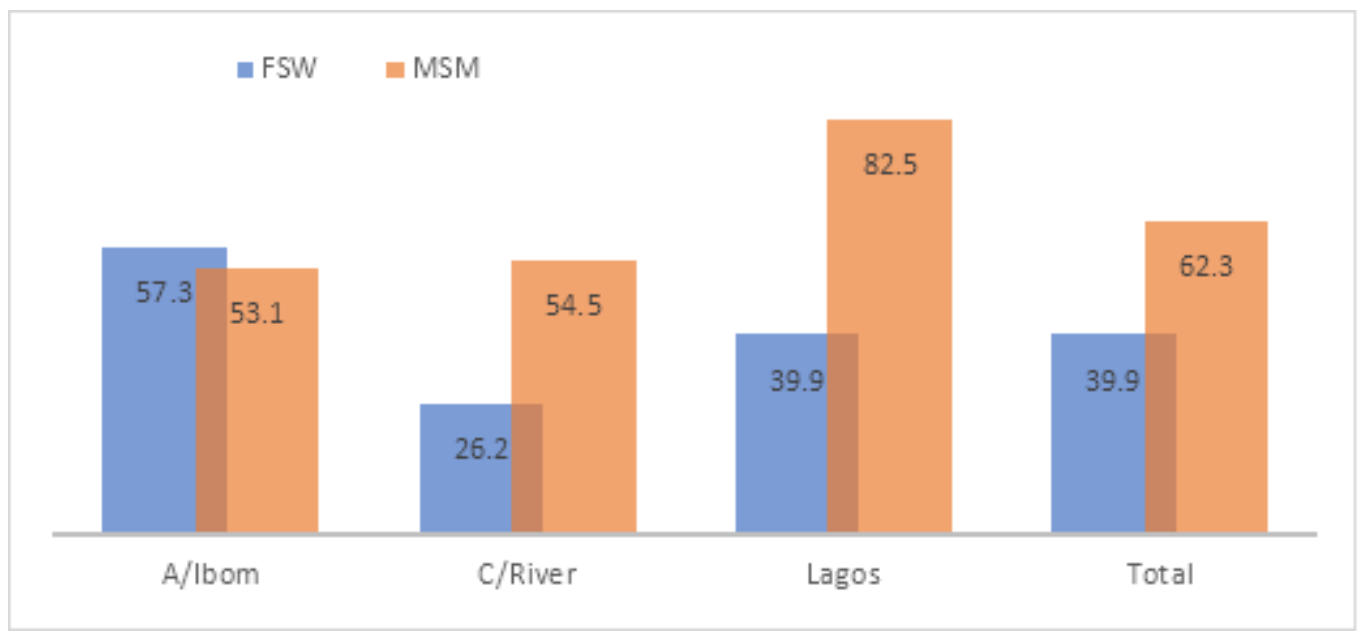

Figure 1

Awareness about PrEP

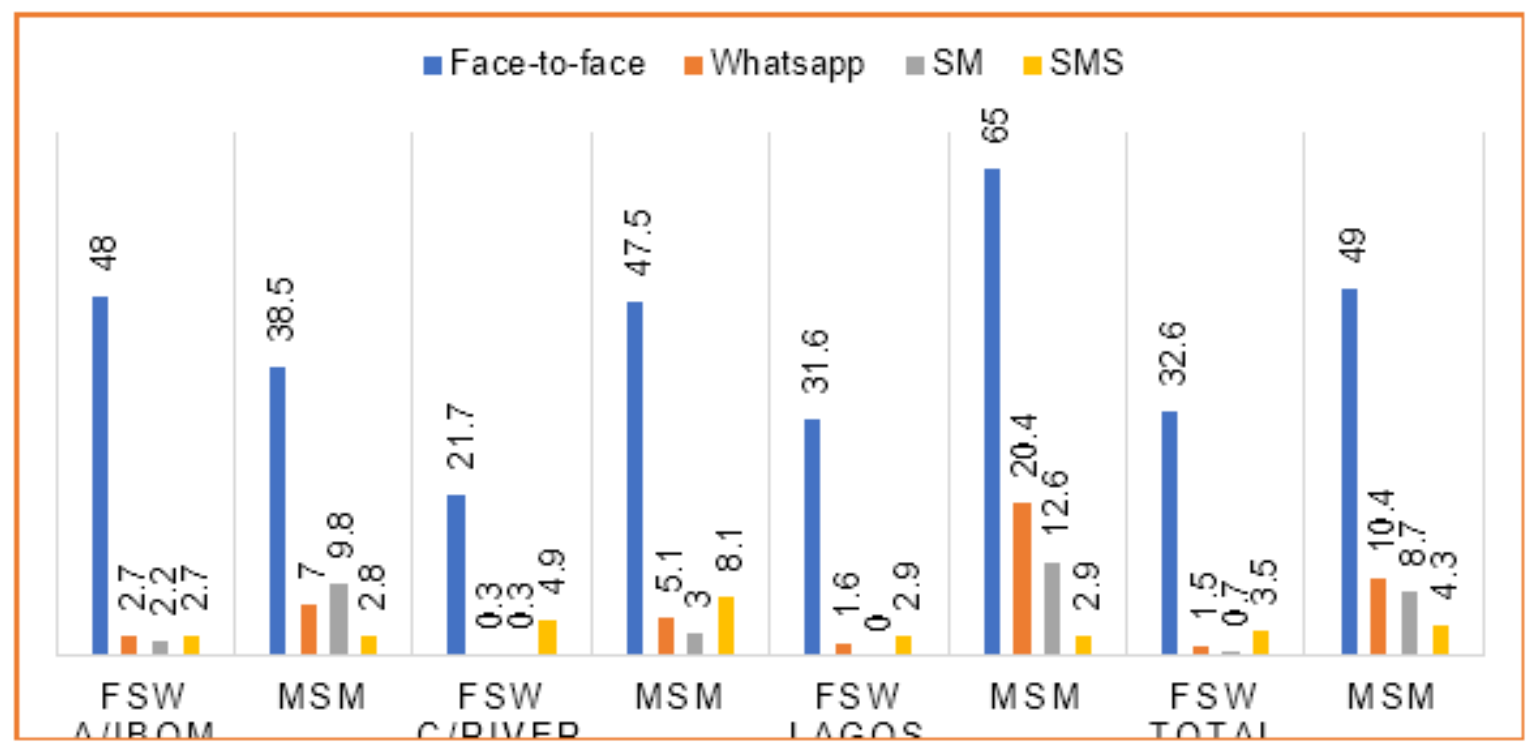

Figure 2

The proportion of reported channel of messaging for PrEP in Nigeria

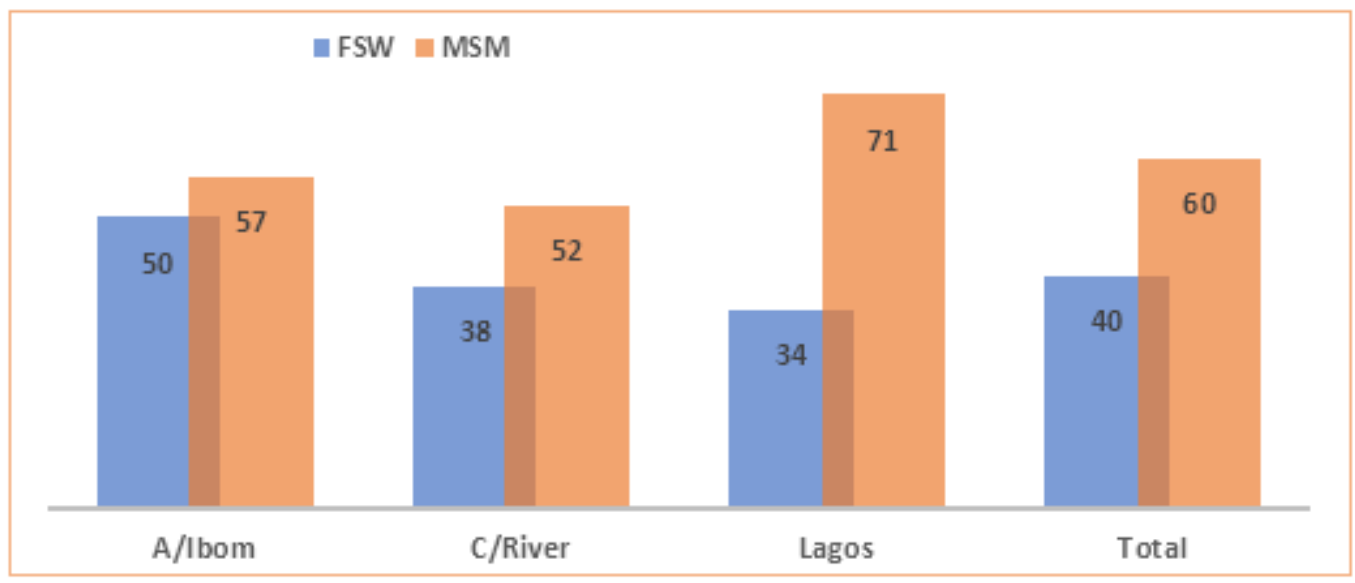

Page $27 / 28$ 
Figure 3

Awareness about HIVST services

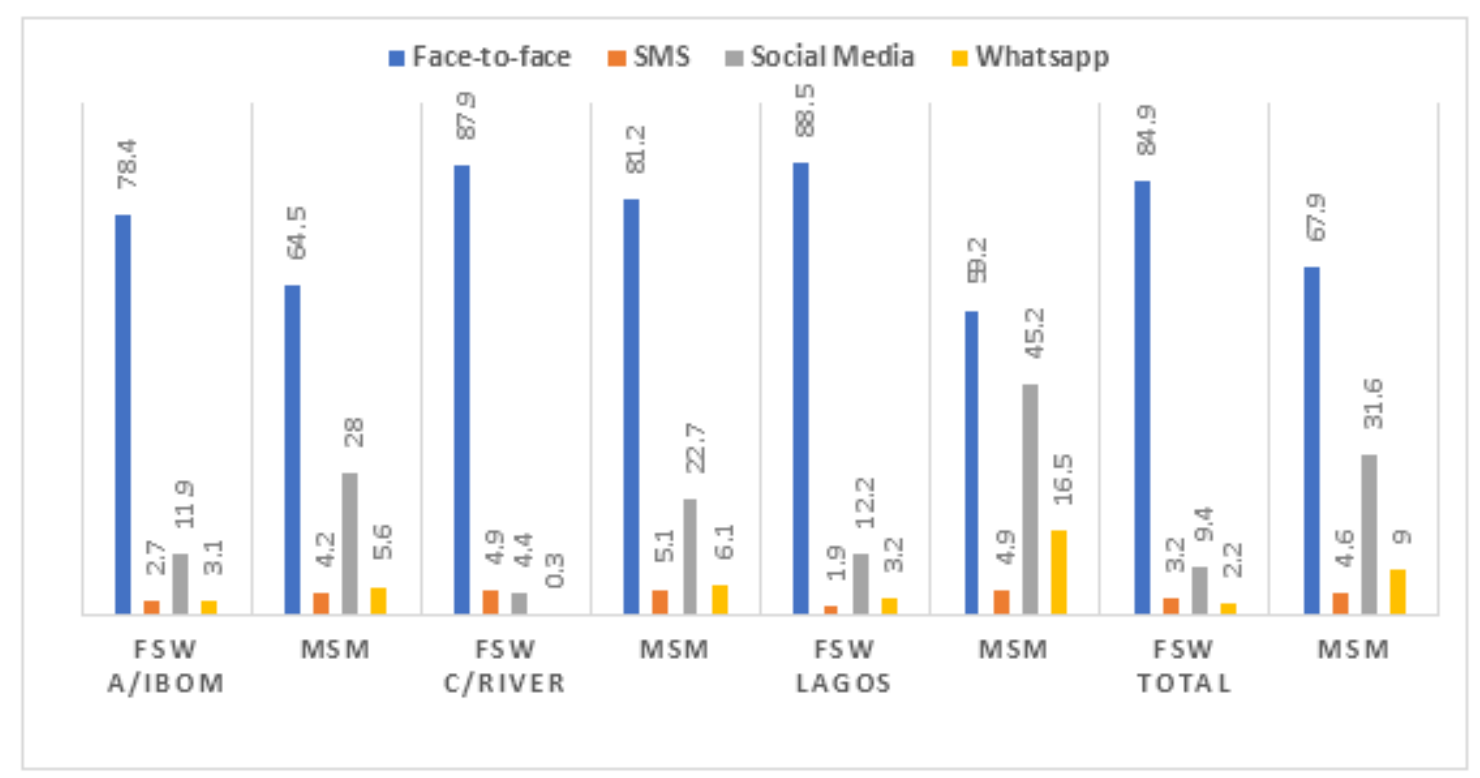

Figure 4

Channel of messaging for HIVST services in Nigeria 